\title{
Strengthening Aboriginal Community Wellbeing
}

\author{
Bronwyn Batten \\ Kellyanne Stanford \\ Office of Environment and Heritage \\ NSW Department of Premier and Cabinet
}

\begin{abstract}
The NSW Government has begun to utilise the concept of 'wellbeing' in relation to Aboriginal communities. This has involved delving into questions about what makes communities strong, and what factors are necessary and/or unique to creating strong Aboriginal communities. This paper provides an insider's view of the government's journey into wellbeing to date, detailing the positions and assumptions that the work began with and, in particular, outlining the process of the creation of the Strengthening Aboriginal Community Wellbeing Framework (the policy context) and the development of a resource for Aboriginal communities - a user friendly software program for communities wishing to holistically assess and plan for strengthening their wellbeing (the 'Strengthening Aboriginal Community Wellbeing Toolkit'). The paper does not delve into critical literature on community engagement and devolution of community control, but rather provides an overview of why and how a particular policy approach was developed which, in the authors' view, will increase community control and power in local decision making and local planning processes.
\end{abstract}

\section{Background}

The concept of wellbeing has been increasingly used in recent times, particularly as it applies to Indigenous communities. For example, in 2002 the NSW Government developed a 10 year plan to improve the wellbeing of NSW Aboriginal people and more recently the Australian Institute of Aboriginal and Torres Strait Islander Studies (AIATSIS) dedicated its 2010 seminar series to the concept of wellbeing. In 2011 the International Journal of Wellbeing was also created to foster interdisciplinary research in the area.

The complexities associated with defining and measuring Indigenous wellbeing are noted in the Commonwealth Government's 2009 Overcoming Indigenous Disadvantage Report (SCRGSP 2009, p. 123). Frameworks that have been developed by government to measure the impact or effect of particular portfolios or programs may not necessarily take into account or measure other interrelated factors that contribute to, or impact upon, wellbeing. For example: government programs that target the reduction of Otitis Media cases within Aboriginal communities may not necessarily note or measure the potential multiple effects and benefits that the reduction of Otitis Media may have on wellbeing - such as an increase in 
school attendance, improved school results or more harmonious family relationships. Yet when trying to develop frameworks that measure impacts in a holistic way, government faces the considerable challenge of developing culturally appropriate and relevant measures of the effectiveness of initiatives.

The NSW Government began exploring the topic of wellbeing in relation to Aboriginal communities and culture and heritage management in 2006 - commissioning a report on how cultural heritage contributes to Aboriginal community wellbeing and how cultural notions of wellbeing can be applied in a policy setting (Grieves 2006). They also conducted a research project examining how Aboriginal promoting and protecting heritage contributes to the health and wellbeing of Aboriginal communities (Carrington \& Young 2011). This research argued that the concept of wellbeing is particularly embraced by Aboriginal communities because it is holistic, and is 'understood to be greater than general happiness, health, welfare or safety' (Carrington \& Young 2011, p.4). Following this initial research, the NSW Government began to explore the application of wellbeing beyond culture and heritage and into the realm of Aboriginal Affairs more broadly.

In 2007, The NSW Parliament Legislative Council Standing Committee on Social Issues commenced an inquiry into overcoming Indigenous disadvantage in NSW. The 2008 report produced by the inquiry contained a number of recommendations which in turn shaped the direction of further work around wellbeing. In 2009, the NSW Government responded to the report and began to develop what they referred to as the Strengthening Community Wellbeing Framework which they envisaged would outlast the usual three year political cycle, assist with defining 'cultural resilience', provide for a process which would assist Aboriginal communities to determine measures of wellbeing and identify local problems and solutions (New South Wales Parliament Legislative Council Standing Committee on Social Issues 2008, pp. xviii - xx).

In addition to the influence of the 2008 inquiry report, the NSW Government's work around Aboriginal wellbeing was guided by state and national policies in Indigenous Affairs at the time. These were: The Council of Australian Governments (COAG) "Closing the Gap”; the COAG “Overcoming Indigenous Disadvantage Indicator Framework”; the NSW State Plan “A new direction for NSW" (2006-2016); and the NSW 10 year Aboriginal Affairs Plan “Two Ways Together” (2002-2012). Whilst highlighting critical areas of importance to 
Aboriginal people, these policies largely adopted a deficit approach - focussing on state or national problems and weaknesses existing in communities. In contrast, by focussing on community wellbeing the approach being developed in NSW allowed for an alternative, strength-based and local approach to Aboriginal Affairs.

In addition to the overriding policy context, the work around wellbeing was also guided by reflecting on the principle community planning initiative of the time: the COAG Trial in the Murdi Paaki region of north-west New South Wales. The Murdi Paaki COAG Trial was one of eight trials being carried out around Australia, with the aim of improving coordination of government services for Indigenous people based on priorities agreed with communities. Whilst the COAG Trial provided and invested substantial financial and human resources to assist communities to develop action plans, no other community planning tools were provided which supported the community to systematically self assess the state of the community, self prioritise the community's desires for change, and then negotiate, develop and monitor implementation of actions plans in partnership with governments and other service providers. From the authors' perspective, a review of NSW and Commonwealth indicator reports demonstrated that, whilst some achievements were made through a variety of Government initiatives, there was clearly an opportunity to improve local Aboriginal community planning approaches and outcomes.

Prior to beginning work on the development of the Aboriginal Community Wellbeing Framework a number of guiding principles were established to govern the work. These included the idea that an Aboriginal wellbeing and community planning approach had to be genuinely holistic - tackling community development from all angles rather than looking at one or two discreet 'problem' areas (e.g. health or education); that Aboriginal disadvantage has its origins in the dispossession and dislocation of Aboriginal people, the accompanying breakdown of community governance and leadership structures, and the suppression of cultural knowledge and practices (including language); and that communities needed tools in order to practically exercise self determination. A key guiding principle was that communities strong in their culture, governance and leadership will have a greater capacity to overcome adversity to identify and achieve their shared goals. Culture, governance and leadership were therefore to be integral elements to the wellbeing work. 
During the initial research around the application of wellbeing beyond heritage and into Aboriginal Affairs broadly (largely conducted in 2008 and 2009) the Commonwealth “Overcoming Indigenous Disadvantage Indicators Report” (SCRGSP 2009, p. 98) highlighted the difficulties in defining and measuring social and emotional wellbeing and identified improvements in data on comparable measures of social and emotional wellbeing of Indigenous people as a priority. Clearly, defining and measuring wellbeing, particularly at the community level, was no easy feat. The NSW Government therefore began to also explore international approaches to both measuring and strengthening community wellbeing, with a particular focus on (but not limited to) processes that targeted Indigenous communities, were holistic and supported a strength-based partnership approach (see Batten 2009 and Batten \& Batten 2011).

Examining approaches to community resilience and wellbeing in Canada further highlighted the principle adopted by the NSW government at the outset that strong culture (including access to Country) was critical to community wellbeing. In Canada research has shown that communities who have strong cultural identity and pride, along with high rates of language retention and self-determination, are far less likely to be affected by mental health issues and suicide and less likely to be in contact with the criminal justice system (Chandler \& Lalonde 1998). Another international example which also informed the NSW work was the “Sustainable Livelihoods Approach” (SLA) and Framework developed and utilised by the UK Department for International Development to alleviate disadvantage. The SLA approach reaffirmed the NSW government's guiding principle that a holistic approach to strengthening community wellbeing was required - one which recognised that a community's wellbeing and its ability to overcome disadvantage was closely associated with a community's access to, and opportunities to use its human, social, natural, physical and financial capital (Department for International Development 1999).

Informed by the research, principles, and policies discussed above, a draft framework identifying factors that were critical to Aboriginal community wellbeing was developed by the NSW Government. The framework drew upon and simplified a range of different, and often overly complex, community strengthening models with adaptations to reflect the unique needs of NSW Aboriginal communities: In particular the framework was underpinned by the importance of cultural strength. A think tank was organised to discuss and further develop the 
draft $^{1}$. Following the think tank, the framework was finalised during workshops held with the Minister for Environment's Aboriginal Cultural Heritage Advisory Committee and in workshops with both the La Perouse and Wellington Aboriginal communities. Both communities were participants in the NSW Governments Two Ways Together Partnership Community Program.

\section{The Strengthening Aboriginal Community Wellbeing Framework}

The Strengthening Aboriginal Community Wellbeing Framework was intended to provide a way to systematically consider a broad range of social, economic, environmental, cultural, governance and service delivery issues that are critical for strengthening and maintaining Aboriginal community wellbeing. Eight interconnected areas were identified as being critically important to wellbeing: cultural identity, access to Country, sense of community, leadership and influence, education and learning, community health and safety, infrastructure

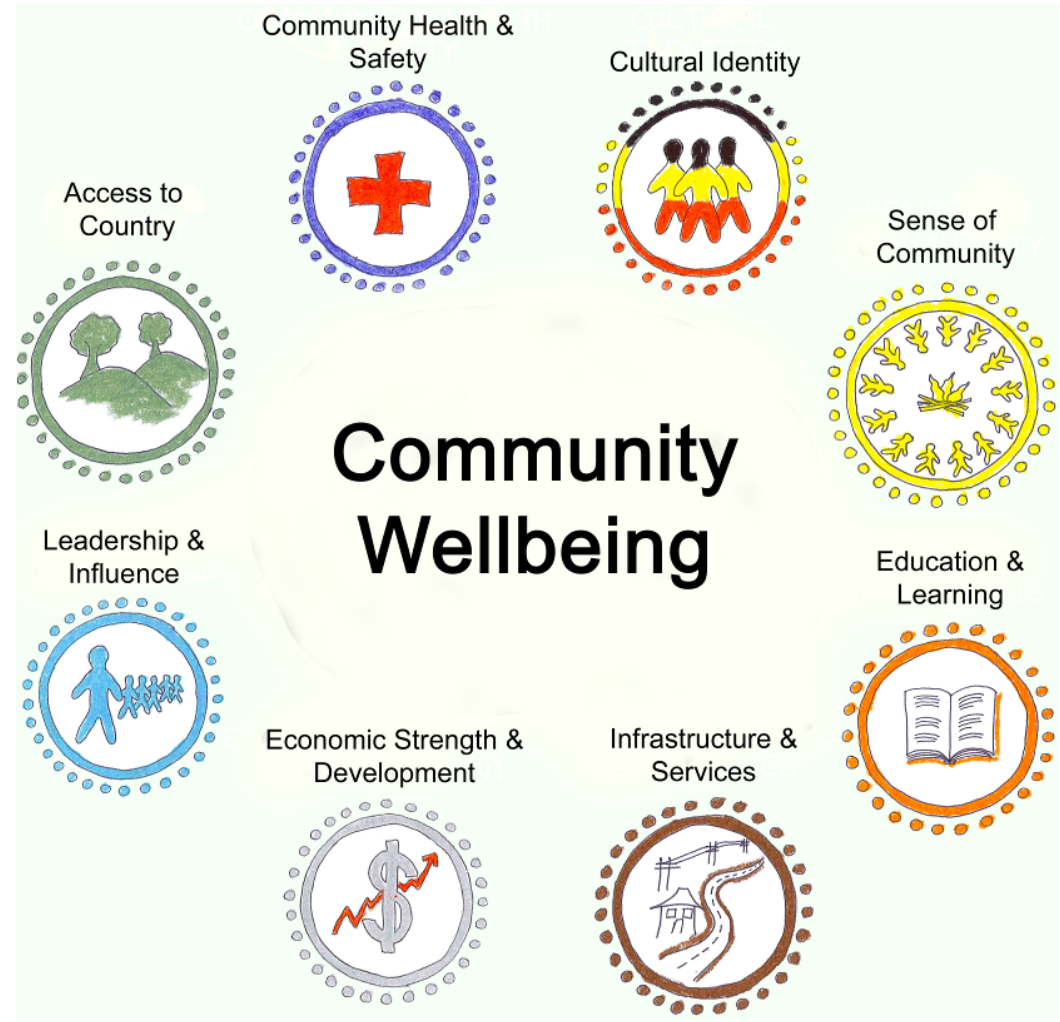

Figure 1: The Strengthening Aboriginal Community Wellbeing Framework

\footnotetext{
${ }^{1}$ The think tank brought together a range of specialists (both Indigenous and non-indigenous) working in the area of wellbeing and community development including academics, individuals well known for their involvement in community development, non-government organisations and representatives from government (including various states and representatives from the Commonwealth Government and an Indigenous researcher working in the field via video link from Canada).
} 
and services, and economic strength and development. The Wellington and La Perouse communities along with Aboriginal advisory bodies provided examples of the types of issues that might be covered under each of the eight areas (providing the 'meat' for the 'bones' of the framework). For example, under the 'access to Country' area, the communities provided the following input:

- Sufficient access to Country provides a sense of belonging - This sense of belonging improves self-esteem, pride in community and pride in one's self.

- For access to be considered adequate, kids in the community must feel a strong connection to Country - This is evidence of the ability to practice culture on Country, share the stories, memories and history that bind people to place.

- Access to Country means provision of education, training and employment opportunities to care for Country - Working on Country is a natural fit for many, but there often aren't enough opportunities.

- Communities want the opportunity to share their land management skills and techniques with the broader community.

- Access to Country is only satisfactory if the community is able to freely practice its culture on its Country (public or private), particularly at sites of significance ${ }^{2}$

- Good partnerships with the broader community facilitate access to Country - When surrounding communities acknowledge and fully understand the importance of access to Country, and work with the indigenous community to allow access and input to management where relevant, access is considered healthy.

- Access to Country may extend to the opportunity to use Country to drive economic development for the community - This means having the freedom to conduct commercial enterprises on Country, and having access to the support and resources to start these enterprises where relevant.

(Office of Environment and Heritage, 2012, p. 35)

The other seven areas that together form the wellbeing framework were similarly 'fleshed out' by the communities. Perspectives gathered on each of the factors contributing to

\footnotetext{
${ }^{2}$ Practicing culture includes hunting, fishing, cultural camps, sharing stories in places of significance. 'Freely practice' means free from onerous rules and regulation.
} 
wellbeing were intended to serve as reference points to prompt other communities using the framework about the range of issues the eight interconnected areas of the framework could cover.

After the development of the framework, the focus of the wellbeing work then turned to developing appropriate processes for measuring wellbeing within the framework. This was to help community and government to track trends in wellbeing over time and improve service delivery in response to trends.

\section{Measuring Wellbeing}

One clear message arising from the 2009 Overcoming Indigenous Disadvantage Key indicators report was that it is difficult to define and measure social and emotional (Indigenous) wellbeing (SCRGSP 2009, p. 123). Australia does not currently have a nationally-agreed approach to defining, measuring or planning for the wellbeing of Aboriginal communities. Existing national and state wellbeing monitoring, reporting and evaluation frameworks have typically been program-specific. There is also a dearth of reliable and conclusive data to establish the solid evidence base required to measure community wellbeing in a holistic way. Several challenges are noted nationally and internationally relating to measurement of Aboriginal wellbeing, including how to bridge the gap between governments’ expectations and Aboriginal peoples’ world views about culturally relevant standards for measuring Aboriginal wellbeing. Governments generally focus on tangible "things" that are easily measured, whilst community understandings of what it means to "be well” often focus on the less tangible dimensions of wellbeing. There are a few examples that challenge this generalisation including work being undertaken in Victoria (Community Indicators Victoria n.d.) and the Australian Bureau of Statistics (2010).

Despite the challenges noted, there is widespread recognition within government that a strong cultural identity and/or sense of community identity is essential to family and community wellbeing and should be measured. There is also recognition within communities and government that the need to measure and track wellbeing over time is critical to improving the circumstances of Aboriginal people. The Overcoming Indigenous Disadvantage report states that 'culture pervades every aspect of the lives of Indigenous people, and some studies have suggested that cultural strength can provide communities with a degree of resilience to entrenched disadvantage' and that 'no single indicator could adequately reflect the place of 
culture in the lives of Indigenous people' (SCRGSP 2009, p. 124). The report highlights the complexities of capturing data that adequately reflects the place of culture in the lives of Indigenous people.

Initially, the approach adopted by the NSW Government to measure wellbeing was to develop a series of indicators that would sit under each of the eight interconnected factors identified as contributing to wellbeing under the Strengthening Aboriginal Community Wellbeing Framework. However, readily available data was largely only available at an aggregated state and national level which meant that the scale of available data was not really meaningful at the community level nor was the available data flexible enough to allow for the diversity of communities situations. In addition to this there was a clear absence of data that could provide a meaningful picture of less tangible or traditionally 'unmeasurable' areas such as a community's cultural strength and identity. The approach therefore evolved to one which aimed to turn arguably the best source of information about Aboriginal communities community held knowledge - into a format that could be used to consistently monitor community wellbeing over time.

It was decided to use the methodology of Appreciative Inquiry to conduct social research with two Aboriginal communities (La Perouse and Wellington) in order to develop a series of statements that communities could utilise to self assess their current levels of wellbeing under each of the areas in the Strengthening Aboriginal Community Wellbeing Framework. Appreciative Inquiry assumes that progress is more likely to be made by focusing on and exploring what we want to see more of (wellbeing), rather than being fixated on studying problems (disadvantage). For this reason, the NSW Government embraced the Appreciative Inquiry method and incorporated it into the toolkit. Appreciative Inquiry was consistent with a key NSW Government principle adopted at the commencement of the wellbeing work that a strength-based wellbeing approach was required. As a method of strengthening wellbeing, Appreciative Inquiry differs from other problem-solving and community planning approaches. The basic assumption of the majority of problem-solving and community planning methodologies is that people and organisations are fundamentally 'broken' and need to be fixed. Problem solving and planning usually involves identifying key problems, analysing root causes, searching for possible solutions, and developing an action plan. In contrast, the underlying assumption of Appreciative Inquiry is that people, organisations and 
communities are full of assets, capabilities, resources, and strengths that are waiting to be located, affirmed, stretched, and encouraged.

Given that Appreciative Inquiry is 1) verbally based, 2) solutions focussed and 3) community or location specific, the NSW government recognised that Appreciative Inquiry could potentially be a great 'cultural match' with Aboriginal communities.

In addition to fleshing out the Strengthening Aboriginal Community Wellbeing Framework the NSW Government began exploring the development of a toolkit to support Aboriginal communities to make informed decisions about their future needs and priorities - decisions grounded in a solid evidence base and a realistic understanding of a community's current situation. The toolkit was intended to identify and support a community's priorities and aspirations and to provide the community with a strong negotiating position when it comes to agreeing on action plans in partnership with government (NSW Government response to the Recommendations of the Legislative Council Social Issues Committee's Inquiry into Overcoming Indigenous Disadvantage 2009, p 13).

Working with both a rural and metropolitan community (along with various Aboriginal advisory bodies with representation across NSW) was a deliberate strategy to help ensure that the diverse needs and views of NSW Aboriginal communities would be addressed throughout the framework and toolkit.

A series of community wellbeing self-assessment statements were developed through the social research by communities telling stories about the best aspects of the past as the foundation for effective visualisation of what would constitute "success" and "wellbeing" in their community for each of the eight areas in the Strengthening Aboriginal Community Wellbeing Framework. As an example, the self-assessment statements produced for the area of ‘access to Country’ are reproduced below:

1. Youth: Younger members of our community feel connected to our Country.

2. Cultural practice: We have the ability to practice culture on our Country, which drives a sense of belonging for members of our community.

3. Partnerships: Our community has strong partnerships with the broader community that allow access to Country and give the community a say where relevant. 
4. Opportunities: There are opportunities for education, training and employment to care for Country for members of our community.

5. Self-determination: Our community has the ability to determine how we use our Country, including for commercial enterprise for the community’s benefit if desired.

(Office of Environment and Heritage 2012, p. 34)

As part of the toolkit process communities are asked to confer on how accurate the statement is for the community and how important it is to the community. A more thorough description of the toolkit processes is included later in the paper within the section 'Development of the Toolkit’.

Throughout the development of the self-assessment statements, the communities also groundtested the framework and agreed that all matters that impacted on their community's wellbeing were addressed under the framework. The communities confirmed the value in having the framework to guide a structured and focussed conversation, with appropriate "prompts” (i.e. the self assessment statements) to facilitate discussion and consideration of a broad range of issues, rather than only focussing on immediately identifiable issues within the community or limiting discussion to those issues that were currently affecting or impacting on their wellbeing.

In the authors' opinion the methodology of Appreciative Inquiry proved to be a great "cultural match" as an approach to consultation about the framework and as a method to hold discussions using the framework. Applying Appreciative Inquiry resulted in meetings that were strength-based and solutions-focussed. It is not uncommon for a strengths-based approach to feel different and unconventional at first. In the authors' observation, after people have experienced this approach they feel much more comfortable about it and can see the logic behind it. As one elder observed, 'We used to tell stories like this. Once upon a time we used to tell stories that made us feel proud. But over the past 20 years it seems all we ever talk about is our problems. We have forgotten how to tell stories differently. This has helped a great deal. It feels right. It is the way we should think about ourselves, our community, our Country’ (Office of Environment and Heritage 2012, p. 27).

\section{The Development of the Toolkit}

Most community strengthening programs have developed methodologies which are contained within lengthy and often complex guide books that require considerable skills and 
background knowledge to guide communities through the process. Many Aboriginal communities feel “over planned”, having been required to go though many community planning processes with government. To address this issue the NSW Government adopted an alternative approach to community assessment and planning: the creation of a user friendly software-based tool designed to support solutions focussed conversations between the community and service providers (both government and non-government) in a way that captures and records that conversation for community planning purposes.

The Strengthening Aboriginal Community Wellbeing Toolkit was developed by tailor making a software program which guided Aboriginal community groups through three important steps to both measure community wellbeing and inform the development of community action plans designed to strengthen community wellbeing.

The steps identified as critical in a community planning process are reflected in the three modules which make up the software:

1 - Assessment - understanding how our community is going

2 - Preparing for negotiation - goal setting and prioritising

3 - Planning together to strengthen wellbeing.

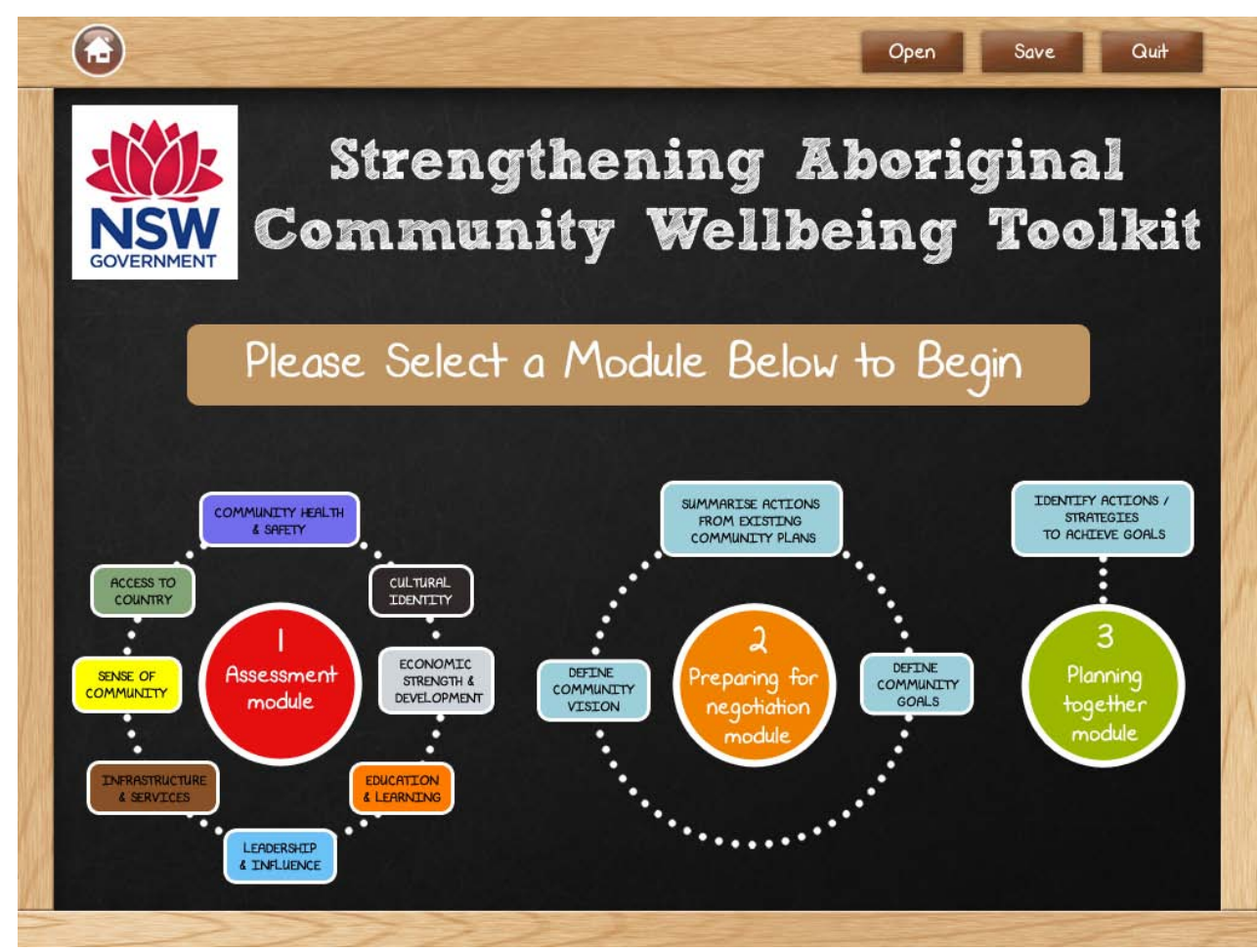

Figure 2: The home screen of the toolkit showing the three different modules 
Module 1 - the assessment module, guides the community through a process of assessing their current levels of wellbeing (utilising the series of community developed self-assessment statements) against each of the eight areas identified as contributing to wellbeing under the Strengthening Aboriginal Community Wellbeing Framework. Communities select answers from a performance and importance ranking for each of the self-assessment statements (48 in total). Figure 3 shows the screen shot of one of the self-assessment statements contained within the area of education and learning. Communities are required to make a judgement on whether they have 'access to well funded and well functioning schools, TAFEs or other educational institutions'. The community must select an answer from the performance criteria from 'always, most of the time, occasionally or never' and also select the level of importance of this particular area to the community 'somewhat important, important, very important, crucial'.

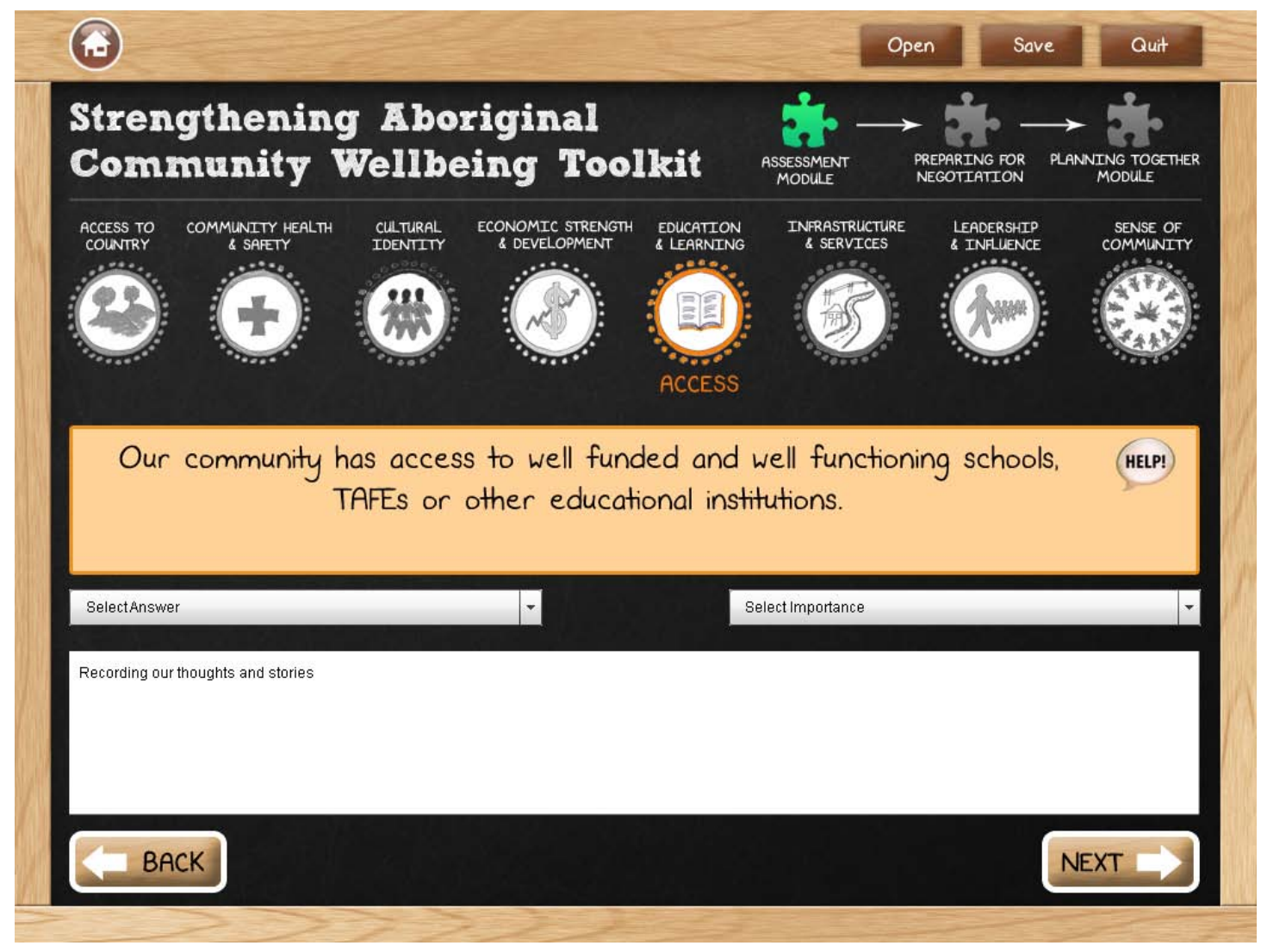

Figure 3: Example of one of the self assessment statements in the assessment module

The toolkit is designed to be used by a group that is representative of the community in 'round table' sessions (this is explored later in the paper within the section 'Who will use the toolkit'). Each of the self-assessment statements would normally involve significant 
discussion by the group, and potentially involve prior consultation with the broader community (for example, surveying the community using the self-assessment statements to gauge broad community perceptions using complimentary, cost effective and user friendly tools such as survey monkey). Module 1 also requires communities to map existing strengths and assets and provides space to record issues and stories for each statement in the assessment module.

Module 2 uses the results of the assessment process to support communities to set and prioritise goals. This step also assists communities to map out their strengths and to incorporate goals and actions from existing community plans.

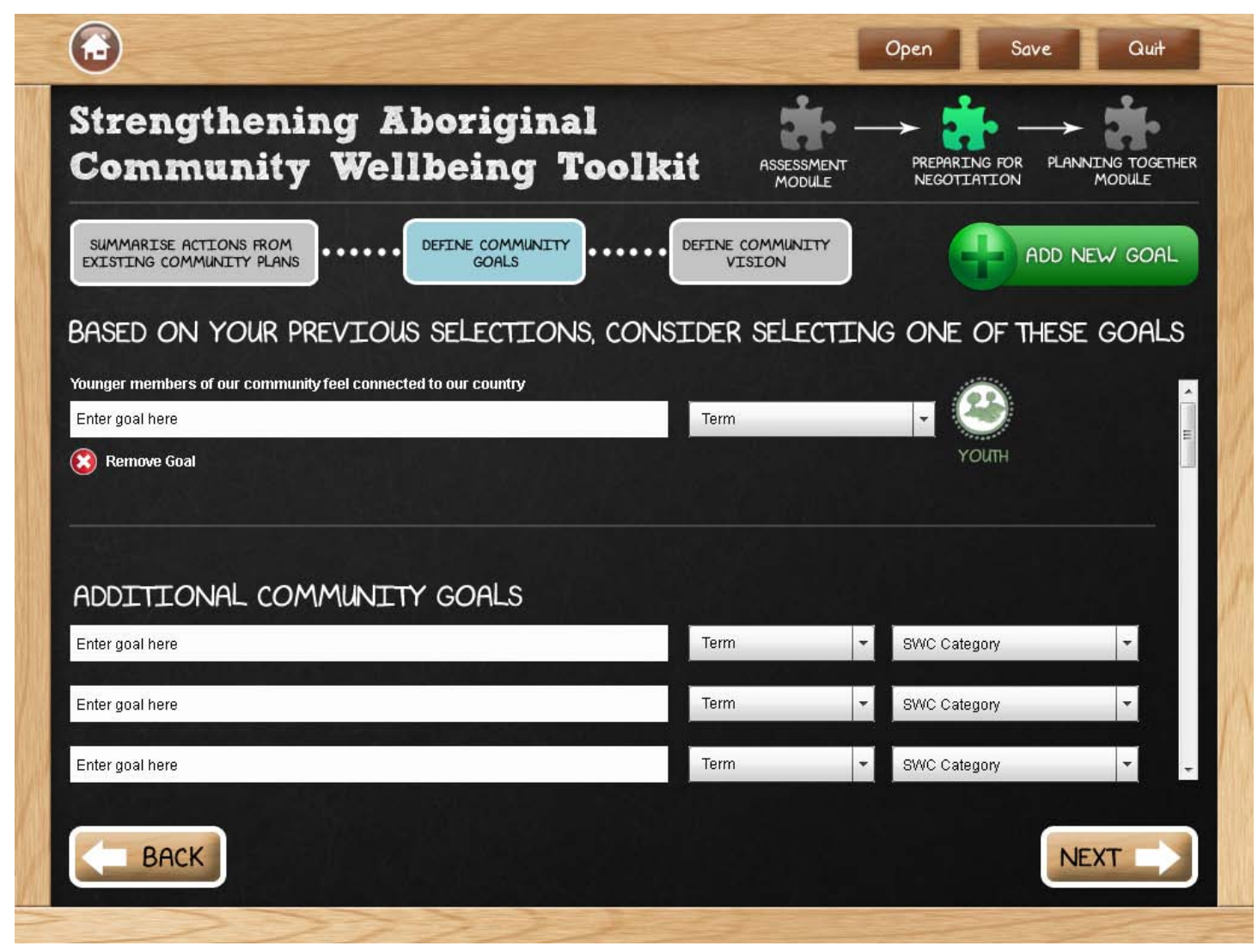

Figure 4: A screen shot from the preparing for negotiation: goal setting and prioritising module

Module 3 uses the results of the assessment and goal setting processes (the two prior modules) to support the community and government to work together to negotiate and plan community and government roles in actions in order to achieve community goals; strengthen 
community wellbeing, and improve service delivery. Relevant non-government organisations and service providers may also be involved.

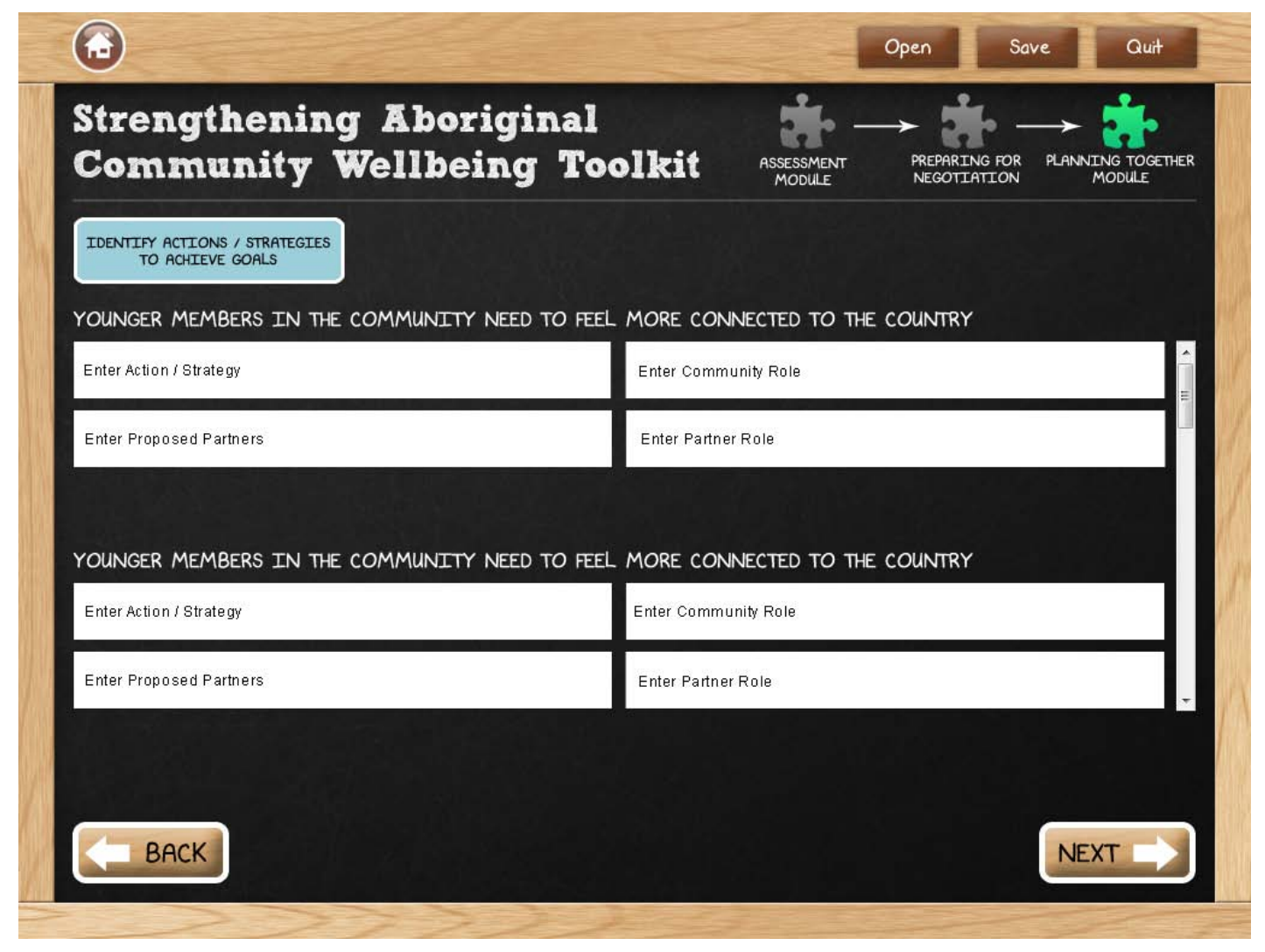

Figure 5: A screen shot from the planning together module

The toolkit provides an output of a report for each of these three stages or 'modules' of the software. The reports can be used to facilitate monitoring of community wellbeing over time and to inform the development of community action plans.

As a result of the overwhelmingly positive feedback from communities about the Appreciative Inquiry methodology, key elements of the methodology were incorporated into the toolkit. For example the toolkit provides a mechanism for capturing the important community conversations, allowing for differing opinions to be recorded .The toolkit also simultaneously provides for recognition of existing community strengths, capacities and assets whilst identifying gaps and aspirations for change. The toolkit has the potential to provide communities with an evidence base about their current level of wellbeing across the areas included within the wellbeing framework. Such an evidence base can assist in negotiations with government about actions and services to address identified gaps and to 
assist with maintaining or strengthening actions, programs and/or services that are critical to their wellbeing. The toolkit also focuses on community perceptions and gives communities a strong say in determining what's right for them.

Consultation with the Wellington and La Perouse Aboriginal communities also highlighted another important consideration for community planning which was incorporated into the toolkit - a process for community prioritisation of issues and actions which was responsive to the community self-assessment process and independent of existing government priorities and/or programs targets.

To support this process a matrix was incorporated into the software which enabled the community to determine for themselves both the level of importance of the issue and the frequency in which it occurred in their community. By considering and ranking both these factors the community was able to utilise the toolkit to simultaneously self-assess and selfprioritise matters affecting their wellbeing. The figure below demonstrates how the software is able to translate the community's answers to help determine priorities for planning. Areas that the community identifies as never or only occasionally occurring in their community, yet are ranked 'crucial' or 'very important' are prioritised highly by the software program, prompting communities to consider focusing their attention on addressing these matters.

\begin{tabular}{|c|c|c|c|c|c|}
\hline \multirow{6}{*}{ 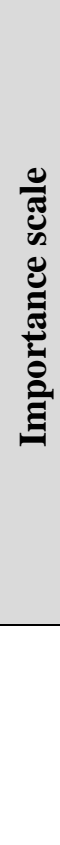 } & $\begin{array}{c}4 \\
\text { Crucial }\end{array}$ & \multirow{2}{*}{\multicolumn{2}{|c|}{ Suggest focusing effort here }} & \multirow{2}{*}{\multicolumn{2}{|c|}{$\begin{array}{c}\text { Maintain current efforts and } \\
\text { consider directing new efforts } \\
\text { here }\end{array}$}} \\
\hline & $\begin{array}{c}3 \\
\text { Very } \\
\text { important }\end{array}$ & & & & \\
\hline & $\begin{array}{c}2 \\
\text { Important }\end{array}$ & \multirow{2}{*}{\multicolumn{2}{|c|}{$\begin{array}{l}\text { Consider maintaining } \\
\text { or redirecting effort }\end{array}$}} & \multirow{2}{*}{\multicolumn{2}{|c|}{$\begin{array}{l}\text { Not a current priority area; } \\
\text { suggest redirecting effort to } \\
\text { higher priorities }\end{array}$}} \\
\hline & $\begin{array}{c}1 \\
\text { Somewhat } \\
\text { important }\end{array}$ & & & & \\
\hline & & $\begin{array}{c}4 \\
\text { Never }\end{array}$ & $\begin{array}{c}3 \\
\text { Occasionally }\end{array}$ & $\begin{array}{l}2 \\
\text { Most of the } \\
\text { time }\end{array}$ & $\begin{array}{c}1 \\
\text { Always }\end{array}$ \\
\hline & & \multicolumn{4}{|c|}{ Performance scale } \\
\hline
\end{tabular}

Figure 6: The Community Self Prioritisation Matrix 
At the end of the assessment process, the community is prompted to identify goals for the areas which the software has suggested should be ranked as 'focus efforts here' and 'maintain efforts here'. The aim is to focus the community efforts and resources on the key actions that will provide the most benefit to their wellbeing. Throughout the community consultation process, communities confirmed that the process of identifying a limited number of focussed key goals and actions will assist with development of a realistically achievable action plan, rather than a comprehensive list of community aspirations. During consultation, communities advised the authors that a long list of aspirations provided a substantial risk of the community being diverted to areas of their wellbeing which were not key community priorities because funding or other type of support for that area may become available rather than focussing on a smaller and more manageable list of areas identified by the community as "key priorities".

However, it is also recognised that community planning may need to legitimately change in order to take advantage of available government funding or new community priorities. Therefore, in order to maintain community autonomy and control over the process, a function was added in to the software, in response to communities' requests, for the option to reject the software suggestions and to have the option to create community goals for areas that the toolkit did not suggest based on community responses alone.

The final module in the toolkit is the 'Planning Together Module'. The aim of this module is to set out the specific actions that will help the community to achieve its goals. Some actions may involve partnerships with government agencies or other organisations, and some actions may be carried out and owned by the community themselves - without the need for partnerships with government or other service providers. This module is designed to be completed with the community working together with the relevant partners so that all parties who will be involved in carrying out an action are also involved in determining what the action is, what their specific roles will be, and what is needed to complete the action. Communities recognised that actions stand a better chance of being completed if they have been negotiated and agreed upon first by all the parties involved rather than spending a lot of time and energy debating how to deliver an action after a plan has already been developed. For this reason, the toolkit provides for development of actions that have been negotiated with and committed to by partners and service providers - rather than finalising the plan in isolation from input from those that will need to respond to the actions. The communities advised that the information generated by the toolkit in the first and second modules (self- 
assessment, prioritisation and goal setting) would place them in a strong position to negotiate appropriate responses from partners and service providers - using information that was self generated, but presented in a way that bridged the "cultural divide” - effectively creating a common language.

\section{Who will use the Toolkit?}

The main users of the toolkit will be communities participating in the NSW Government Partnership Community Program. The Government intends that the Partnership Community Program will establish ‘a basis for Aboriginal communities and government (State and Federal) to work together to improve outcomes for Aboriginal people through partnership, mutual accountability and strengthened local decision making' (Department of Aboriginal Affairs 2009a). The program aims to 'improve service delivery and outcomes on the ground for Aboriginal people; and strengthen Aboriginal community wellbeing' (Department of Aboriginal Affairs 2009a). The Partnership Community program is based on the principle that 'Aboriginal people know best the needs of their communities, and that government agencies and Aboriginal communities need to work in partnership to improve outcomes on the ground' (Department of Aboriginal Affairs 2009a).

Community Program officers employed by Aboriginal Affairs NSW (previously called the Department of Aboriginal Affairs) have been trained by the Office of Environment and Heritage in facilitating the use of the toolkit in communities. The toolkit is designed to be used by a community engagement group as defined in the Partnership Community Governance Framework (Department of Aboriginal Affairs 2009b). However, the toolkit is also available as a resource for communities, peak bodies and other interested parties who may not currently be participating in the Partnership Community Program. The toolkit is designed to be user friendly and intuitive; nevertheless a manual is also available as an additional resource to help any community who wishes to use the toolkit. In order for the toolkit to be an effective resource for communities not participating in the Partnership Community Program, it is recommended that the toolkit is used by an engagement group or equivalent - i.e. a group that: reflects the diversity of the community; is representative of the interests of the entire community, and is supported to make decisions on behalf of the community. 


\section{Discussion}

The toolkit has the potential to meet dual aspirations, those of the community e.g. facilitating community self determination and improving community wellbeing and those of government e.g. achieving real progress in community wellbeing and meeting program and strategy targets and outcomes.

\section{Meeting Community Aspirations}

The communities involved in developing and using the toolkit have provided informal feedback to staff involved in developing the toolkit that it will support self determination by enabling community self assessment - communities will tell their perspective instead of being told about themselves by government using government collected data. Government information still remains a useful source of information but having the community "story" is important - discrepancies between the two "stories" will prompt a useful and valuable discussion between government and communities. The communities involved in the development of the toolkit also believed that the fact that it allows for self prioritisation of community priorities instead of priorities being forced by accessing available funds and programs due to government created Indigenous targets also contributes to community selfdetermination.

When using the toolkit, the community is generating their own information (turning information held in and by the community - information that they own and control) into a form that is compatible with government processes. The community is making its needs and wants heard in a way that is mutually understood by both community and government. Communities have advised that negotiating with service providers utilising community generated data will be a first for many communities and will be empowering and "level out the playing field”. Communities have also advised that they have felt disempowered in past negotiations due to lack of "evidence" to support their priorities and their inability to demonstrate a community process for developing priorities.

The communities involved in the development of the toolkit felt that the toolkit supported a strength-based participatory community self assessment and planning process. The toolkit commences the community discussion in a positive way- encouraging the community to reflect upon and recognise the good things (assets and capacities - both tangible and intangible) in the community. In the authors' observation it is often easier to agree upon the 
positives which encourages a consensus building process and lays the foundation for application of positive lessons from the past along with aspects of an Appreciative Inquiry methodology throughout the 3 modules of the toolkit. The toolkit is strength-based and solution-focussed. The communities consulted acknowledged that the process of recognising existing community strengths, assets and capacities is one quite often overlooked in planning processes and discussions with government. In the authors opinion past community planning processes generally commence with "problems and issues”, and without a holistic assessment of the current status of the community. However in the "wellbeing" process, the consultation commenced with discussions about existing community strengths and assets and moved into a conversation about what the community would like to see more of - through this process, the community identified key matters that warranted focussed action, and prepared to plan, in partnership with Government and other service provider, to address those matters,

Some members of the Wellington and La Perouse communities expressed the feeling that the toolkit will help establish processes where for the first time community planning provides an opportunity to tell their own stories in their own voices, and to assess, for themselves, their own wellbeing. The wellbeing framework enables the community to genuinely consider its wellbeing independent of government plans and targets or other governance or statutory reporting requirements. The community is not forced to slot its information into a format that suits the latest government plan. It is a resource that can outlive political cycles and 'state plans' and so on.

\section{Meeting Government Aspirations}

The NSW Government Ombudsman’s 2011 report ‘Addressing Aboriginal disadvantage: the need to do things differently' observed that the answer to addressing disadvantage is not providing further financial resources to initiatives, rather a different approach to the way governments plan, fund and deliver services.

The report identified five key areas for change:

- Aboriginal leadership

- Improving the capacity to respond to vulnerable Aboriginal children and adolescents

- Investing in education

- Building economic capacity in Aboriginal communities 
- Establishing a new accountability framework for addressing Aboriginal disadvantage

(NSW Ombudsman, 2011)

The toolkit and framework has the potential to support a different way of working with Aboriginal communities and the five areas identified by the Ombudsman, and more, by supporting Government and community to work together to identify ways to plan, fund and deliver services. In addition, the Strengthening Community Wellbeing Framework enables the Government to demonstrate actions that are responsive to community identified needs and aspirations. The framework could also be utilised to develop portfolio and program specific indicators which could be aggregated to a state level whilst providing for comparison at the community level (given it forms the basis of community planning).

The Strengthening Community Wellbeing Framework and Toolkit supports a number of recommendations within the Auditor General's performance audit of the NSW Aboriginal Affairs Plan (2003 - 2012) - Two Ways Together. These include:

- the Partnership Community Program has merit and should be supported by providing ongoing training for the community governance bodies;

- that a process be developed which monitors NSW Government compliance with the requirement to work the community governance bodies within the Partnership Community Program;

- that Aboriginal people know best the needs and aspirations of their local communities;

(NSW Auditor-General, 2011)

The toolkit does this by serving as a resource to Partnership Communities (and any other community that wishes to use it) that will help prepare them to engage with government to jointly agree on a series of actions to best meet community goals and priorities. The toolkit will also contribute to changing the current way of implementing a plan in Aboriginal communities by getting the community involved early in assessment, prioritisation and planning stage; informing both the community and Government decision making process; and more clearly defining what the community actually wants and how that will contribute to improving wellbeing. 
The NSW Government's commitment to strengthening community wellbeing was clearly indicated in 2010 with bipartisan support to amend the NSW Constitution in order to recognise and acknowledge Aboriginal people of NSW. Hansard recorded the NSW Government's pledge to close the gap in Aboriginal disadvantage, and to work with nongovernment organisations and the community to improve Aboriginal wellbeing (New South Wales Parliament, 2010, pp. 26125 - 26133, pp. 26152 - 26158).

\section{Conclusion}

The Strengthening Aboriginal Community Wellbeing Toolkit was formally launched on 14 March 2012 by the Minister for Environment and Heritage. It is still therefore very much “early days" for the toolkit. The use and effectiveness of the toolkit will be monitored and then reviewed two years after its launch and the Office of Environment and Heritage will be asking anyone involved in using the toolkit to contribute to its review so that it can continue to be refined to meet the needs of communities. The evaluation will address a broad spectrum of issues from user friendliness to its usefulness as a tool for both measuring wellbeing and facilitating community planning. A survey has been set up to help collect information on the effectiveness of the toolkit whilst the experience of using the toolkit is fresh in the minds of communities.

The comprehensive evaluation of the effectiveness of the wellbeing research, framework and toolkit will need to begin with a definition of the measure of success. The evolution of the wellbeing research into the development of a framework and toolkit has occurred simultaneously within a changing Aboriginal affairs policy context in NSW - from Two Ways Together to Closing the Gap and now to the development of a new (and yet unknown) policy framework. The NSW Government is currently developing a new Aboriginal Affairs Strategy, which may result in indicators and targets developed for the state level. The question remains as to how communities will measure the success of the framework and toolkit at the community level. It is important that the evaluation of the framework and toolkit take into account the success of this dual focus - meeting both community and government aspirations - at the local level.

The La-Perouse community became the first community to formally use the framework and toolkit with the La-Perouse Aboriginal Community Alliance utilising it on the 22 March 2012 in a forum of approximately 50 people. The use of the framework and toolkit by the La- 
Perouse Community in a large community setting demonstrates that its use is flexible - one size does not fit all. Given the large number of people present and the challenges of reaching consensus in a forum of that size the La Perouse community did not use the toolkit software, instead they utilised the toolkit handbook and the appreciative enquiry methodology to inform a community forum about the strengths of the La Perouse Community within the context of the framework and to hold round table sessions using the toolkit self assessment statements. A second session utilising the framework will also occur in order to determine what the community would like to see more of, and how they think this would best occur (i.e. developing community derived solutions). The La Perouse Community Alliance (a team of approximately 15 people) will then analyse the results of the community forum and utilise the toolkit software in order to generate a community action plan in partnership with services providers.

The toolkit and framework are not intended to be a "magic wand" in Aboriginal Affairs. The toolkit and framework will not solve all issues in communities overnight nor will they immediately bridge the gap between the government's and communities' understandings of issues affecting community wellbeing - it is hoped, however, that over time these resources will create dialogue and discussion around wellbeing in communities and how to actively improve it and empower communities to turn their knowledge and perceptions into information to inform community planning. This will allow communities to be in a position of informed strength and to be able to negotiate relevant actions with government and other service providers to achieve their goals.

At this stage the toolkit is only available via a CD for use on a local computer and not online. This decision has been made deliberately, and in consultation with the Aboriginal communities that assisted with development of the toolkit. Although promoting and distributing the toolkit online would be much easier, and the ease of access for many communities could be greater, distributing the toolkit via CD ensures that all information entered into the software program remains the property of communities. No information is stored online by government, only on community computers.

The wellbeing work began its journey by simply undertaking an exercise to "define" Aboriginal community wellbeing in order to measure improvements as a result of government investment and commitment. As discussed throughout this paper, this journey 
and task evolved into a far more complex task - that of supporting communities to have an increased level of control over defining, assessing, planning for, and measuring, their own wellbeing, in a culturally appropriate way, and in a way that was easily understood by government, facilitated an appropriate government response and enabled the government to track and measure the success of investments into service delivery and policy responses.

A copy of the toolkit on CD can be obtained by contacting the Office of Environment and Heritage - Country, Culture and Heritage Division on (02) 95856453 or emailing aboriginal.affairs@environment.nsw.gov.au.

\section{References}

Australian Bureau of Statistics, 2010. 4703.0 - Framework for Measuring Wellbeing: Aboriginal and Torres Strait Islander Peoples, 2010. Available online: http://www.abs.gov.au/ausstats/abs@.nsf/mf/4703.0?OpenDocument

Batten, B. 2009. Building Aboriginal Community Resilience: Learning from Canadian Experiences, unpublished report.

Batten, B. and Batten, P. 2011, 'Strengthening Aboriginal community wellbeing - learning from Canadian experiences’, Journal of Australian Indigenous Issues, vol. 14, no. 4, pp. 85-100.

Carrington, B. \& Young, P. 2011, Aboriginal Heritage and Wellbeing, Department of Environment, Climate Change and Water (NSW), Sydney South. Available online: http://www.environment.nsw.gov.au/nswcultureheritage/AboriginalHeritageWellbeing. $\underline{\mathrm{htm}}$

Chandler, M. \& Lalonde, C. 1998, 'Cultural Continuity as a Hedge against Suicide in Canada's First Nations', Transcultural Psychiatry, vol. 35, no. 2, pp. 191-219.

Community Indicators Victoria n.d, An Introduction to Community Indicators Victoria, Community Indicators Victoria. Available online: http://www.communityindicators.net.au/about_us

Department of Aboriginal Affairs (NSW) 2009a, Partnership Community Program Fact Sheet, Available online: http://www.daa.nsw.gov.au/data/files//PartnershipCommProg_factsheet110811.pdf

Department of Aboriginal Affairs (NSW) 2009b, Draft Partnership Community Governance Framework, Available online: http://www.daa.nsw.gov.au/publications/TWTPCGF.pdf

Department for International Development 1999, Sustainable Livelihoods Guidance Sheets: Introduction, DFID, London, Available online: http://www.eldis.org/vfile/upload/1/document/0901/section1.pdf

Grieves, V. 2006, Indigenous Wellbeing: A framework for Governments’ Aboriginal Cultural Heritage Activities, Department of Environment and Conservation (NSW). Available online:

http://www.environment.nsw.gov.au/resources/cultureheritage/GrievesReport2006.pdf 
New South Wales Auditor - General 2011, Performance Audit, Two Ways Together - NSW Aboriginal Affairs Plan. Available online:

http://www.audit.nsw.gov.au/ArticleDocuments/143/213_Two_Way_Together_Aborigi nal_Affairs.pdf.aspx

NSW Ombudsman 2011, Addressing Aboriginal disadvantage: the need to do things differently. Available online:

http://www.ombo.nsw.gov.au/publication/PDF/specialreport/SR_Aboriginal\%20disadv antage\%20report.pdf

New South Wales Parliament 2010, Legislative Council, Parliamentary Debates, (Hansard) (October 19). Available online:

http://www.parliament.nsw.gov.au/Prod/parlment/hanstrans.nsf/V3ByKey/LC2010101 9/\$File/541LC215.pdf

New South Wales Parliament Legislative Council Standing Committee on Social Issues 2008, Overcoming Indigenous disadvantage in New South Wales. Final report (Report 41) NSW Parliament, Sydney, NSW. Available online: http://www.parliament.nsw.gov.au/Prod/parlment/committee.nsf/0/5e06cb564943b260c a25750e0002d05d/\$FILE/081125\%20Final\%20Report\%20for\%20website.pdf

New South Wales Parliament Legislative Council Standing Committee on Social Issues 2009, New South Wales Whole of Government Response to the Recommendations of the Legislative Council Social Issues Committee's Inquiry into Overcoming Indigenous Disadvantage.

http://www.parliament.nsw.gov.au/Prod/parlment/committee.nsf/0/5e06cb564943b260c a25750e0002d05d/\$FILE/090527\%20Govt\%20response\%20to\%20OID\%20final\%20re port.pdf

Office of Environment and Heritage 2012, Strengthening Aboriginal Community Wellbeing Toolkit User Manual, Office of Environment and Heritage, NSW Department of Premier and Cabinet, Sydney South.

SCRGSP (Steering Committee for the Review of Government Service Provision). 2009, Overcoming Indigenous Disadvantage: Key Indicators 2009, Productivity Commission, Canberra. Available online: http://www.pc.gov.au/_data/assets/pdf_file/0003/90129/key-indicators-2009.pdf 